III Simpósio Paranaense de Modelagem.

Simulaçäoe Controle de Processos

Artigo:

39

ISSN : 1984-7521

Páginas: 286-292

\title{
MÓDULO EXPERIMENTAL - TANQUE AQUECIDO, SINTONIA DO CONTROLADOR
}

\author{
Victor Hugo da Silva Barlatti ${ }^{1 *}$, Roberto Navarro Koepsel ${ }^{2}$, Cid M. G. Andrade ${ }^{2}$, Marcos de Souza \\ 1 - Universidade Estadual de Maringá - UEM - PR, victor_barlatti@hotmail.com \\ 2 - Universidade Estadual de Maringá - UEM - PR
}

\begin{abstract}
Resumo - Os sistemas de controle são cada vez mais necessários nas indústrias, a importância de controlar um processo pode ser vista claramente em suas vantagens (redução de custos, aumento da segurança do processo, uso mais eficiente de matérias-primas e energia, especificações de qualidade mais rigorosas, impacto ambiental reduzido), nesse sentido a utilização de módulos experimentais tem possibilitado simular processos e formas de controle. Este trabalho apresenta o estudo da obtenção da função de transferência de um módulo experimental de controle de temperatura desenvolvido na UEM, bem como a utilização de um controlador PID através de uma interface desenvolvida no software Scilab, e a sua sintonia utilizando os métodos de Ziegler e Nichols. Foi obtida uma modelagem matemática conforme a literatura. Não foram obtidos resultados satisfatórios com o controlador sintonizado. Conclui-se portanto que o módulo experimental apresentou uma modelagem matemática conforme a literatura, porém não apresentou a resposta esperada quando utilizado o controlador PID e os métodos de sintonia de Ziegler e Nichols. Esta divergência na curva de resposta do controlador se deve a limitações físicas do sistema de aquecimento e/ou o método de sintonia não foi adequado ao sistema.
\end{abstract}

Keywords: Controle. Módulo experimental. Tanque aquecido. Controle PID. Ziegler e Nichols. Função de transferência. Controle de temperatura.

\section{Introdução}

Os sistemas de controle estão ficando cada dia mais imprescindíveis nas indústrias, que procuram cada vez mais aprimorar seus processos com o objetivo de otimizar a produtividade e reduzir os custos. A importância de controlar um processo pode ser vista claramente em suas vantagens como além da redução de custos também o aumento da segurança do processo, o uso mais eficiente de matérias-primas e energia, especificações de qualidade mais rigorosas, impacto ambiental reduzido [1].

Um sistema de controle fundamental em diversos processos industriais é o controle de temperatura, em especial o controle de temperatura de líquido em um tanque o qual é a abordagem deste trabalho.

A finalidade de um sistema de controle de temperatura de um tanque é aquecer o líquido contido no tanque de forma que o mesmo saia em uma temperatura desejada. $\mathrm{O}$ sistema opera em malha fechada, ou controle por realimentação, para garantir que a temperatura desejada seja obtida com precisão [2].

O controle por realimentação se caracteriza pelo fato de a variável de saída ser comparada continuamente com a referência, que é a própria variável de entrada, a fim de detectar possíveis desvios da resposta do sistema, em relação à referência [3].

Para a correção das variações no sistema é necessário utilizar um algoritmo de controle, e o mais utilizado em sistemas de controle industrial em todo o mundo, devido ao seu desempenho, funcionalidade e facilidade de sintonia, é o controlador PID (ProporcionalIntegral-Derivativo), que consiste em três coeficientes que são variados de forma a obter a melhor resposta [4].

O controlador PID possui a função de transferência dada pela Eq. 1[4,5].

$$
G_{C}(s)=K_{p}+\frac{K_{i}}{s}+K_{d} s
$$


Onde $K_{p}$ é o ganho proporcional, $K_{i}$ é o ganho integral e $K_{d}$ é o ganho derivativo.

Ziegler e Nichols, em 1942, propuseram dois métodos matematicamente simples para a sintonia do controlador PID, que são utilizados até hoje [4]. Neste artigo será considerado o primeiro método.

O método consiste em encontrar uma função de transferência na forma da Eq. 2 e utilizar os parâmetros encontrados para a obtenção da Tabela 1 [6].

$$
G(s)=\frac{K}{\tau s+1} e^{-s L}
$$

Onde K é o ganho do sistema, $\tau$ é a constante de tempo e L é o atraso.

Tabela 1 - Constantes do controlador PID pelo método de Ziegler e Nichols.

\begin{tabular}{|c|c|c|c|}
\hline Controlador & $K_{p}$ & $K_{i}$ & $K_{d}$ \\
\hline $\mathrm{P}$ & $\frac{\tau}{L}$ & - & - \\
\hline $\mathrm{PI}$ & $0.9 \frac{\tau}{L}$ & $\frac{0.3 K_{p}}{L}$ & $0.5 L K_{p}$ \\
\hline $\mathrm{PID}$ & $1.2 \frac{\tau}{L}$ & $\frac{0.5 K_{p}}{L}$ & \\
\hline
\end{tabular}

O objetivo deste trabalho é obter a função de transferência de um módulo experimental de controle de temperatura desenvolvido no Departamento de Engenharia Química da Universidade Estadual de Maringá, sintonizar um controlador PID usando as regras de Ziegler e Nichols e obter a curva de resposta esperada.

\section{Materiais e métodos}

O módulo experimental utilizado consiste de um tanque em acrílico transparente de formato cilíndrico, com uma entrada de água e uma saída de vazão constante através de uma bomba com vazão de $5 \mathrm{~L} / \mathrm{h}$.

O sistema de aquecimento do tanque é composto através de uma resistência elétrica de $6800 \mathrm{~W}$, tensão $220 \mathrm{~V}$, ligada a um triac BTA41 e um acoplador óptico MOC 3020, o sensor de temperatura empregado é o LM35.

Foi utilizado o Arduino Uno para fazer toda a interface entre o sensor de temperatura, o sistema de aquecimento e o software Scilab, onde foi programado o controlador e criado uma interface gráfica onde o operador pode definir o set point, o tipo de controle e suas constantes.

Um sinal PWM (Pulse-Width Modulation ou modulação por largura de pulso), controlado pelo Scilab, é enviado pelo Arduino Uno ao MOC o qual faz o controle de disparo do triac, controlando assim a potência do sistema de aquecimento.

As Fig. 1a e Fig. 1b demonstram, respectivamente, o módulo experimental e o esquema do sistema de controle, a Fig. 2 apresenta a interface gráfica do controlador. 


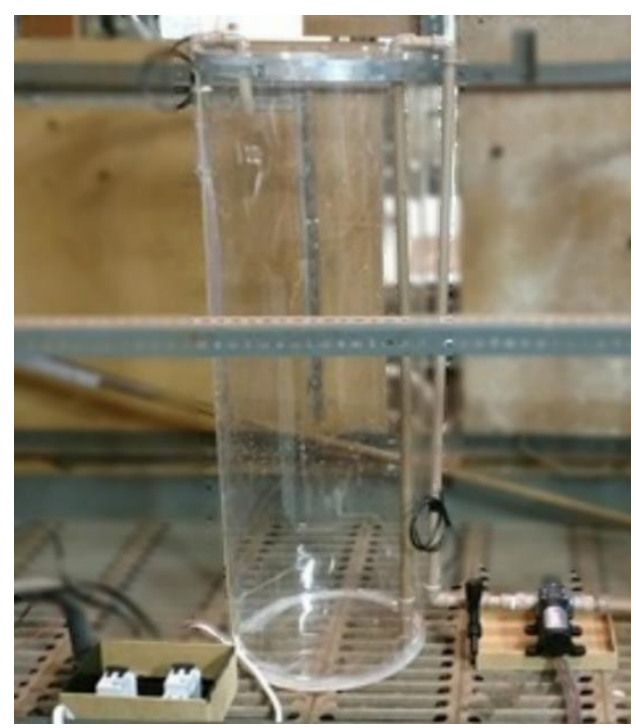

a

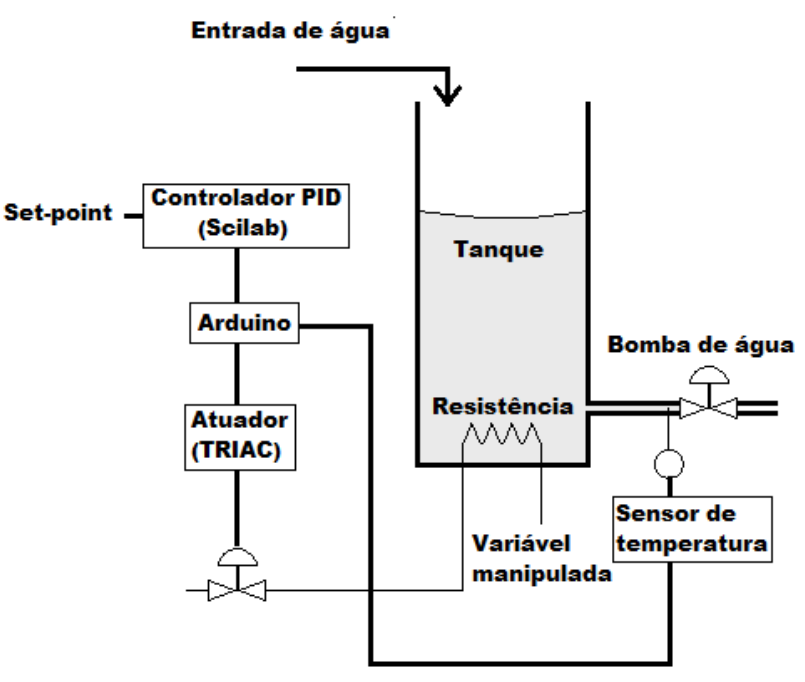

b

Figura 1 - (a): Módulo experimental; (b): Esquema do sistema de controle.

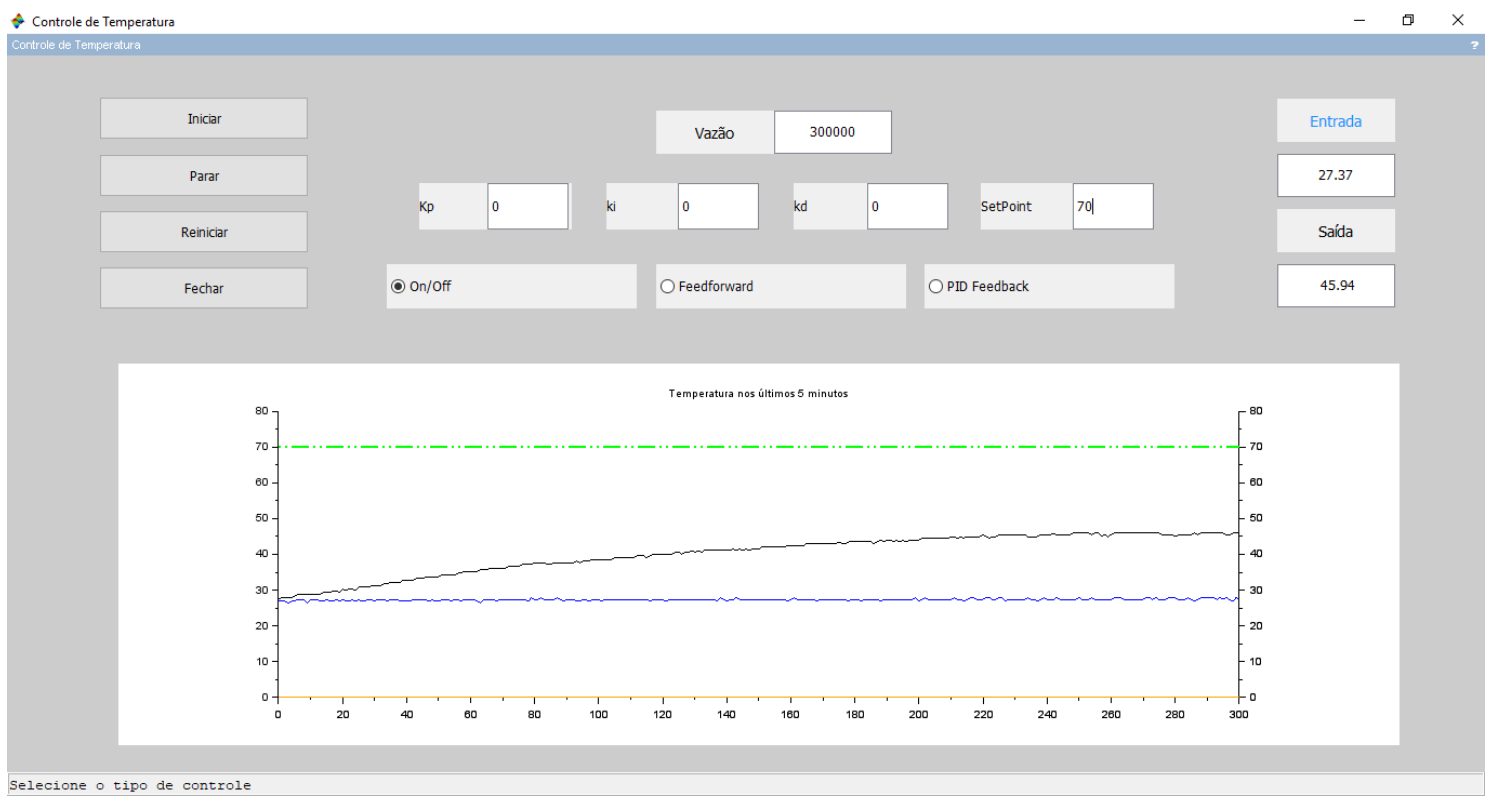

Figura 2 - Interface gráfica do controlador.

O tanque foi preenchido com água até uma certa altura que resultou em um volume de 17,45 litros, mantendo uma vazão de entrada e saída constantes, esse volume permaneceu constante. A partir dessas características físicas do sistema foi calculada a função de transferência teórica do sistema, dada pela Eq. 3, através do modelo fenomenológico [1].

$$
G(s)=\frac{0.0029}{202 s+1}
$$


Através de um ensaio em malha aberta, onde foram dados dois degraus, de 40 e $80 \%$ respectivamente, na potência do aquecedor do sistema, pode-se observar a resposta da temperatura de saída do tanque (Fig. 3) e assim foi possível caracterizar uma função de transferência experimental do processo.

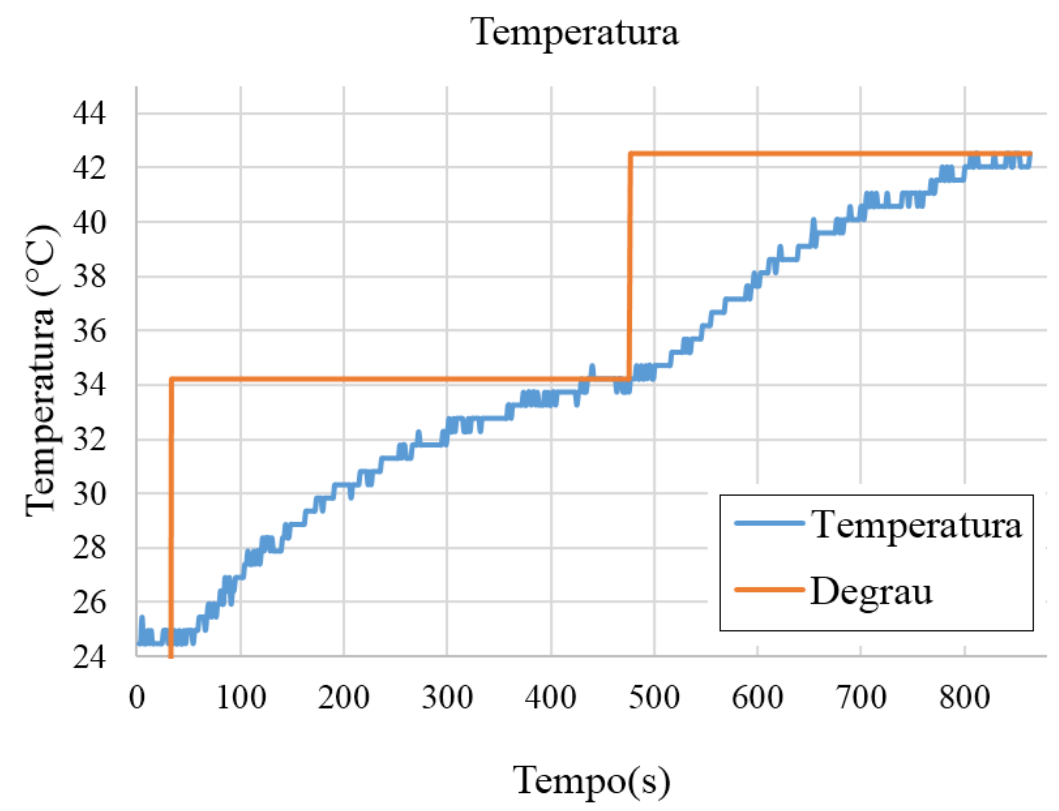

Figura 3 - Resposta ao degrau da temperatura de saída.

Como pode ser observado na Fig. 3, o sistema apresenta um tempo morto significativo, portanto foram utilizados os métodos de identificação para sistemas FOPDT (First-Order Plus Dead-Time) descritos em [6], a função de transferência experimental encontrada do processo é dada pela Eq. 4.

$$
G(s)=\frac{0.003}{180 s+1} e^{-10 s}
$$

Com a função de transferência experimental, foi feita a sintonia do controlador utilizando o primeiro método de Ziegler e Nichols,

Com os valores das constantes do controlador PID obtidos, Tabela 2, foram inseridos no controlador através da interface gráfica e foram feitos novos ensaios, dessa vez através de uma mudança no set-point.

Tabela 2 - Constantes do controlador PID obtidas pelo método de Ziegler e Nichols.

\begin{tabular}{|c|c|c|c|}
\hline Controlador & $K_{p}$ & $K_{i}$ & $K_{d}$ \\
\hline $\mathrm{P}$ & 18 & - & - \\
\hline $\mathrm{PI}$ & 16.2 & 0.486 & 108 \\
\hline $\mathrm{PID}$ & 21.6 & 1.08 & \\
\hline
\end{tabular}




\section{Resultados e Discussão}

A Fig. 4 apresenta a curva de resposta obtida pelo controlador sintonizado considerando um set-point de $31{ }^{\circ} \mathrm{C}$. A Fig. 5 apresenta a atuação do controlador.

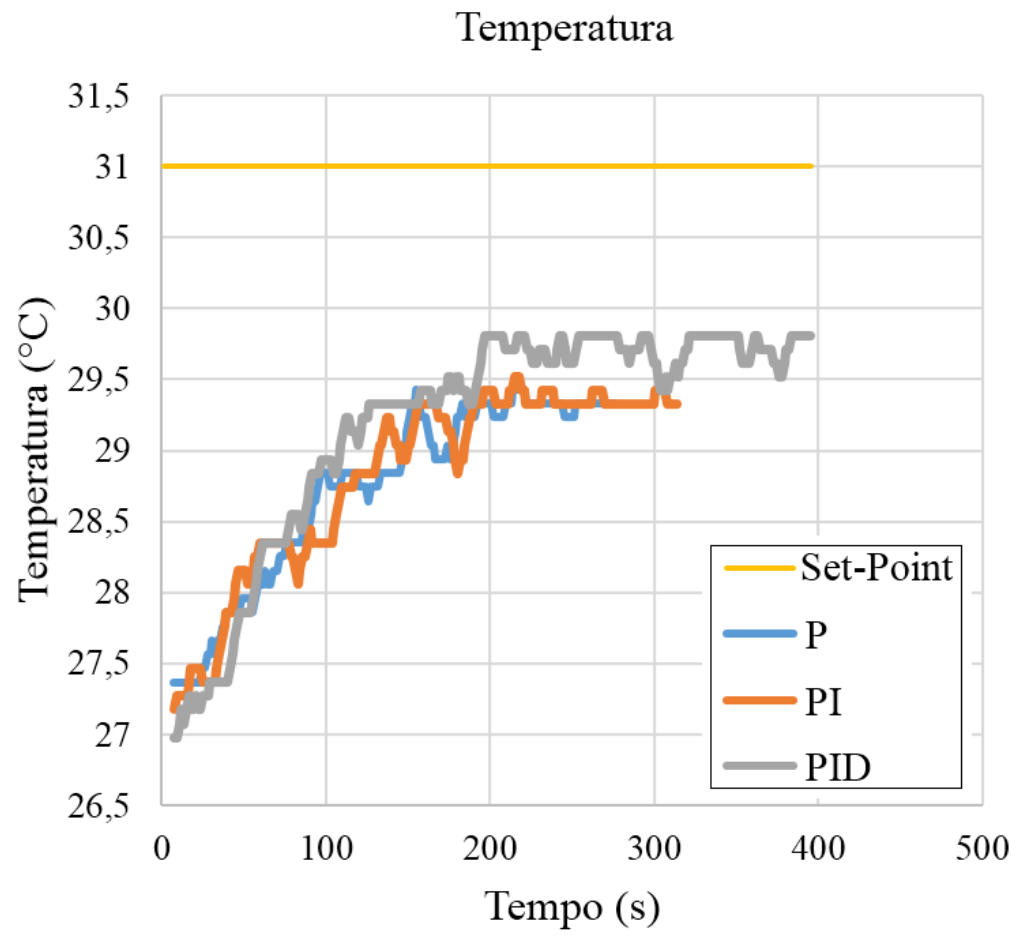

Figura 4 - Curva de resposta obtida pelo controlador sintonizado

Ação de Controle

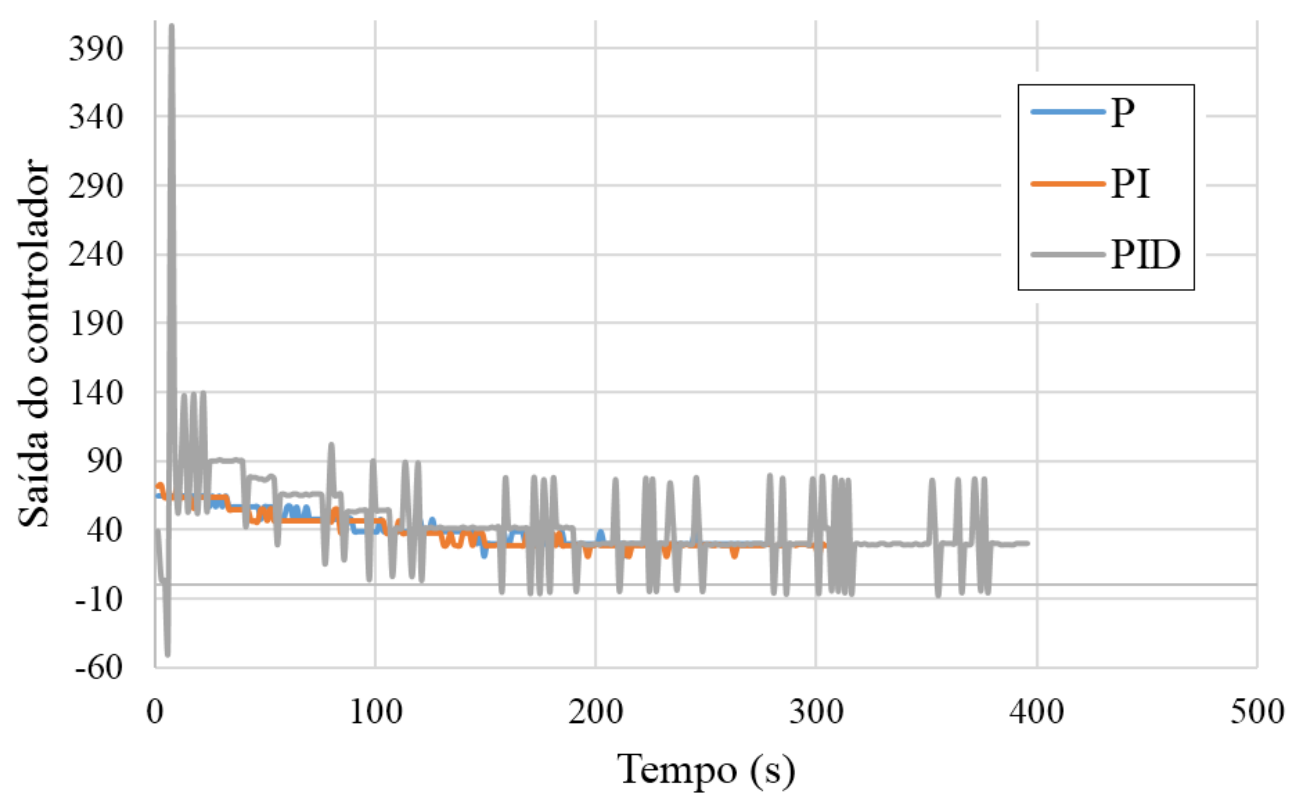

Figura 5 - Atuação do controlador sintonizado. 
Analisando a Fig. 4 observa-se que a resposta obtida não se mostrou conforme o esperado de acordo com a literatura, visto que a temperatura de saída não atingiu o set point estabelecido para os controladores PI e PID.

A Fig. 5 nota-se que as ações de controle P e PI não tiveram variações bruscas, o que mostra uma atividade de controle estável, já a ação de controle PID teve grandes variações, comprovando a significância da ação derivativa, mas não apresenta uma ação de controle suave.

Também foi possível observar que a temperatura lida pelo sensor LM35 apresenta algumas variações, isso se deve a sua baixa precisão, visto que é um sensor de baixo custo, diante do sistema.

Como os resultados não foram completamente satisfatórios, obtêm-se algumas hipóteses: a variável manipulada (resistência elétrica) pode não apresentar potência suficiente para o sistema, sugere-se então aumentar a potência da resistência ou diminuir a vazão do tanque ou o volume de água; o método de sintonia do controlador utilizado pode não ser adequado para o sistema, sugere-se então o estudo de outros métodos de sintonia do controlador.

\section{Conclusão}

Neste trabalho obteve-se a função de transferência de um módulo experimental de controle de temperatura desenvolvido no Departamento de Engenharia Química da Universidade Estadual de Maringá e implementou-se um controlador PID através de uma interface gráfica desenvolvida no Scilab, e realizou-se também a sua sintonia utilizando os métodos de Ziegler e Nichols.

O trabalho mostrou que o módulo experimental apresenta uma modelagem matemática conforme a literatura, porém não apresentou a resposta esperada quando utilizado o controlador PID e os métodos de sintonia de Ziegler e Nichols. Esta divergência na curva de resposta do controlador se deve a limitações físicas do sistema de aquecimento e/ou o método de sintonia não foi adequado ao sistema.

\section{Agradecimentos}

Este trabalho agradece ao PEQ-UEM e a Capes pela bolsa de mestrado.

\section{Referências}

1. COUGHANOWR, Donald R.; LEBLANC, Steven E.. Process Systems Analysis and Control. 3. ed. New York: Mcgraw-hill, 2009.

2. JIE, Lee Ming. PID IMPLEMENTATION OF UMP MINI AUTOMATION PLANT: PART 1 - HEATING TANK. 2010. 93 f. TCC (Graduação), University Malaysia Pahang, 2010.

3. MAYA, Paulo Alvaro; LEONARD, Fabrizio. Controle Essencial. 2. ed. São Paulo: Pearson, 2014.

4. SANTOS, Carla M. M. dos et al. DESENVOLVIMENTO DE UM MODULO DE CONTROLE DE NIVEL UTILIZANDO O KIT ARDUINO UNO. Anais do XX Congresso Brasileiro de Automática. Belo Horizonte, 2014. 
5. NISE, Norman S.. Engenharia de Sistemas de Controle. 6. ed. Rio de Janeiro: Ltc, 2012.

6. COELHO, Antônio Augusto Rodrigues; COELHO, Leandro dos Santos. IDENTIFICAÇÃO DE SISTEMAS DINÂMICOS LINEARES. Florianópolis: Editora da Ufsc, 2004. 